\title{
CONSTRAINTS ON THE WIND STRUCTURE OF OB STARS FROM THEORETICAL He II LINES
}

\author{
Roberto O. J. Venero, Lydia S. Cidale, ${ }^{1}$ and Adela E. Ringuelet ${ }^{1}$ \\ Facultad de Ciencias Astronómicas y Geofísicas, Universidad Nacional de La Plata, Paseo del Bosque S/N, \\ 1900 La Plata, Buenos Aires, Argentina; roberto@fcaglp.unlp.edu.ar \\ Received 2001 November 12; accepted 2002 June 20
}

\begin{abstract}
Theoretical profiles of He II lines in OB stars with an expanding, spherically symmetric atmosphere are computed. The extended atmospheric model is formed by a classical photosphere, characterized by the effective temperature and the surface gravity, and superimposed layers that have different velocity and temperature structure. We solve rigorously the radiative transfer equation, simultaneously with the statistical equilibrium equations for multilevel atoms, by making use of Feautrier's method in the comoving frame. We discuss the influence on the He II lines of the hydrodynamic and thermodynamic structure of the atmosphere, paying special attention to those configurations that give rise to emission lines. The main conclusions of our work can be summarized as follows:
\end{abstract}

1. The shape of the profiles is determined by the velocity gradient at the base of the wind whenever a positive temperature gradient occurs.

2. In O-type stars, the emission-line intensity depends quite sensitively on $\log g$, in agreement with the Walborn luminosity criterion.

3. In addition, we are able to produce emission and absorption profiles that are in qualitative agreement with those observed in $\mathrm{O}$ and $\mathrm{B}$ stars.

4. We also confirm the previous Cidale \& Ringuelet and Venero, Cidale, \& Ringuelet results that showed that a warm, extended, and rapidly expanding atmosphere is sufficient to give rise to emission components in the line profiles.

Subject headings: line: formation — radiative transfer — stars: atmospheres — stars: early-type

\section{INTRODUCTION}

The $\mathrm{H}$ and $\mathrm{He}$ II lines provide a valuable tool to investigate the structure and physical state of early-type stars. The OB-type stars with emission lines usually display a wide variety of line profiles, such as single or double emissions or P Cygni profiles, that reveal the presence of extended atmospheres with outward-accelerating winds.

One of the most analyzed lines in the optical region is He II $\lambda 4686$ (3-4). A single emission peak flanked by broad emission wings is the usual description of this line in most Of stars (Grady, Snow, \& Timothy 1983), although simple P Cygni profiles with a shallow absorption component have also been reported (Conti \& Leep 1974; Grady et al. 1983). A small group of stars (e.g., $\lambda$ Cep and $\zeta$ Pup) exhibit more complex profiles composed of two emission features flanking a self-reversed absorption, like a Beals P Cygni type III profile (Beals 1951; Mihalas \& Conti 1980). Such profiles are also observed in $\mathrm{H} \alpha$ (Heap 1971; Conti \& Niemela 1976) and He II $\lambda 10124$ (Mihalas \& Lockwood 1972). The He II $\lambda 4686$ line can present quasi- or nonperiodic variations in some Of and $\mathrm{O}(\mathrm{f})$ stars, and changes in emission amplitude, as well as in FWHM and in the profile structure, have been observed. Quasi-periodic variations in this line are attributed to rotational modulation in a stellar wind, as in the case of the O8 If star HD 192639 (Rauw et al. 2001), while the nonperiodic variability is consistent with changes in the mass-loss rate, in the wind ionization balance, or even in the velocity field (Grady et al. 1983). He II $\lambda 4686$ is also consid-

\footnotetext{
${ }^{1}$ Member of the Carrera del Investigador Científico, CONICET.
}

ered a luminosity indicator in O stars (Walborn 1971; Conti \& Leep 1974): as the luminosity increases, the absorption line profile gradually turns into a complete emission profile.

In spite of the difficulty of observing the shape of the wings in the line of He II $\lambda 3203$ (3-5), mainly due to the presence of an ozone atmospheric band (Morrison 1975), this line seems to appear in absorption in most OB stars, even though He II $\lambda 4686$ is in strong emission.

Another relevant transition of He II is $\lambda 1640$ (2-3), which is present in the UV spectrum of stars earlier than B2 and disappears in the later types (Peters 1990). This line often displays an absorption profile, but it can also present emission components in early Of supergiants, while P Cygni profiles are observed in spectral types earlier than O6f (Walborn \& Nichols-Bohlin 1987). Some authors have suggested that the many Of stars showing strong emission in this line can be considered a link between Of stars and WN7 objects (Willis \& Stickland 1980; Nandy et al. 1980).

From a theoretical point of view, the wide variety of He II line profiles observed among OB stars can be attributed to different conditions in the atmospheres, such as asymmetries, velocity fields, temperature stratification, and massloss rate.

In order to reproduce the observed lines in early-type stars, many calculations have been performed, improving either the atomic model or the atmospheric structure. The relevance of the non-LTE (NLTE) effects in early-type stars was shown by Auer \& Mihalas (1972), who obtained a good quantitative agreement with the absorption line spectra of $\mathrm{H}$, He I, and $\mathrm{He}$ II for normal $\mathrm{O}$ stars with a static, planeparallel photospheric model. However, the atmospheres of stars with emission lines are quite different from those that 
correspond to plane-parallel standard models; their structures are more complex, because they are dominated not only by NLTE effects but also by the wind dynamics. Calculations of $\mathrm{He}$ II emission lines have been performed by Klein \& Castor (1978), Mihalas \& Kunasz (1978), Kunasz (1980), Hamann \& Schmutz (1987), Hillier (1987), Gabler et al. (1989), Santolaya-Rey, Puls, \& Herrero (1997), and Herrero, Puls, \& Villamariz (2000), with nonstatic, spherically symmetric models. Most of these models computed NLTE transitions of He II for a wind driven by radiation forces and a temperature structure in radiative equilibrium.

In the last years, the modeling of the physical conditions of stellar atmospheres has been focused on the hypothesis of a wind driven by radiation forces, the CAK (Castor, Abbott, \& Klein 1975) or modified CAK (Pauldrach, Puls, \& Kudritzki 1986) theory. These models have allowed us to advance in the understanding of early-type stars, giving important tools to obtain their fundamental parameters. The "unified" models for linedriven winds (Puls et al. 1996) derive the wind momentum-luminosity relationship, allowing us to determine the distance to far hot stars through the fitting of their observed spectral features.

Using standard unified models, Herrero et al. (2000) show that while most of the computed transitions are in good agreement with the observed lines, He II $\lambda 4686$ always presents large discrepancies. These authors show that the fits to $\lambda 4686$ are improved by setting the He II resonance lines in detailed balance or by artificially adding a $\rho^{2}$ dependent background opacity that simulates line blocking.

The existence of a density effect on the He II lines was also shown some years ago by Kunasz (1980), who found that the line profiles are very sensitive to changes in density. Adopting smaller densities than those resulting from stellar winds driven by radiation forces, he was able to obtain a configuration with He II $\lambda 4686$ in emission and He II $\lambda 3203$ in absorption. Otherwise, from the study of IR excesses, Castor \& Simon (1983) have concluded that runs of density and velocity are not homologous in all $\mathrm{O}$ star winds, revealing large variations in atmospheric structure from star to star.

These results suggest that some stars could present density and velocity distributions different from those derived with the CAK theory. If this were the case, one would expect that density or velocity effects become evident in $\mathrm{H}$ and $\mathrm{He}$ II line profiles, since they are sensitive to the velocity gradients at the base of the wind (Cidale \& Ringuelet 1993; Venero, Cidale, \& Ringuelet 1999). It would also be plausible that the expected temperature rise in the outer atmospheric layers as a consequence of NLTE departures (Cayrel's mechanism) could be further increased by the existence of nonradiative fluxes.

We suggest that the discrepancies in the fits of the spectral features could reveal the existence of a wind structure that differs from that of CAK theory. This structure would originate from the contributions of other terms in the momentum and energy equations, e.g., magnetic fields (Mihalas \& Conti 1980), nonthermal equilibrium fluctuations of a photosphere (Cannon \& Thomas 1977; Andriesse 1979), and rotation (Petrenz \& Puls 2000). It is significant that early-type stars with nearly the same spectral type and luminosity display line profiles with quite different appearances. This fact, which leads to an intricate spectral classification $[\mathrm{O}, \mathrm{Oe}, \mathrm{Of}, \mathrm{O}(\mathrm{f}), \mathrm{Ofnp}$, etc.], might be due to the existence of different wind accelerating mechanisms or temperature structures.

Few theoretical investigations have been focused on the effects of departures from radiative equilibrium on the spectra of early-type stars. Perhaps this is due to the fact that the optical lines, traditionally used in the spectral analysis, are rather insensitive to temperature variations in the extended atmosphere. For instance, the $\mathrm{H} \alpha$ emission profile is insensitive to temperature variations in a nonradiative temperature structure in A- and B-type stars (Catala \& Kunasz 1987; Cidale \& Ringuelet 1993). However, the intensity of IR H lines $\mathrm{Pa} \beta, \mathrm{Pa} \gamma$, and $\mathrm{Br} \gamma$ varies strongly under changes of the temperature-law parameters (Cidale et al. 2000). It is also found that free-free emission produced by a hot region placed close to the photosphere contributes to the enhancement of the near- and far-IR continuum flux and has no effects on the near-UV and visible continua (Vázquez, Cidale, \& Ringuelet 1993).

The purpose of the present paper is to explore the significance of alternative atmospheric conditions (temperature and velocity gradients) in the line-forming regions of $\mathrm{OB}$ stars for the appearance of the He II lines. At present, we do not have a self-consistent treatment for the momentum and energy equations to investigate these alternative cases, so we are restricted to proposing ad hoc distributions to describe the wind expansion and the temperature distribution throughout the stellar atmosphere. These proposed distributions are represented by schematic models with few free parameters in a fine grid of effective temperatures and effective gravities. The main goal is to reproduce the most relevant characteristics of the observed profiles, to be able to establish the proper conditions in the stellar atmospheres.

In $\S 2$, we present the adopted atmospheric models. In $\S 3$, we briefly refer to the radiative transfer problem and to the atomic model. The results obtained are presented in $\S 4$ and the discussion and general remarks in $\S 5$; finally, the comparison with the observations and the conclusions are given in $\S \S 6$ and 7, respectively.

\section{WIND MODEL}

In order to compute the He II line profiles, we model the extended atmosphere as a homogeneous, spherically symmetric, nonrotating, steady wind, with solar abundances for all the chemical elements. The main absorption sources for the continuous radiation are bound-free and free-free transitions of atoms and ions of $\mathrm{H}, \mathrm{He}, \mathrm{C}, \mathrm{N}, \mathrm{O}, \mathrm{Ne}$, and $\mathrm{Si}$ and Thomson scattering; all of them are treated with their corresponding occupational numbers in LTE.

The wind velocity distribution starts in the photosphere with subsonic velocities and increases monotonically with radius until it reaches an asymptotic value (with high velocities). We designate as $R_{\mathrm{ph}}$ the photospheric radius, i.e., the radius at which the temperature reaches the effective temperature value, and the variable $r$ is in units of $R_{\mathrm{ph}}$.

Besides the " $\beta$ law" that was widely studied in model atmospheres of W-R and O stars,

$$
V(r)=V_{0}+\left(V_{\infty}-V_{0}\right)\left(1-\frac{1}{r}\right)^{\beta},
$$

where $V_{\infty}$ and $\beta$ are free parameters, we also explore the following expression, which describes flows with a low-velocity regime at the base of the wind that abruptly accelerates in 


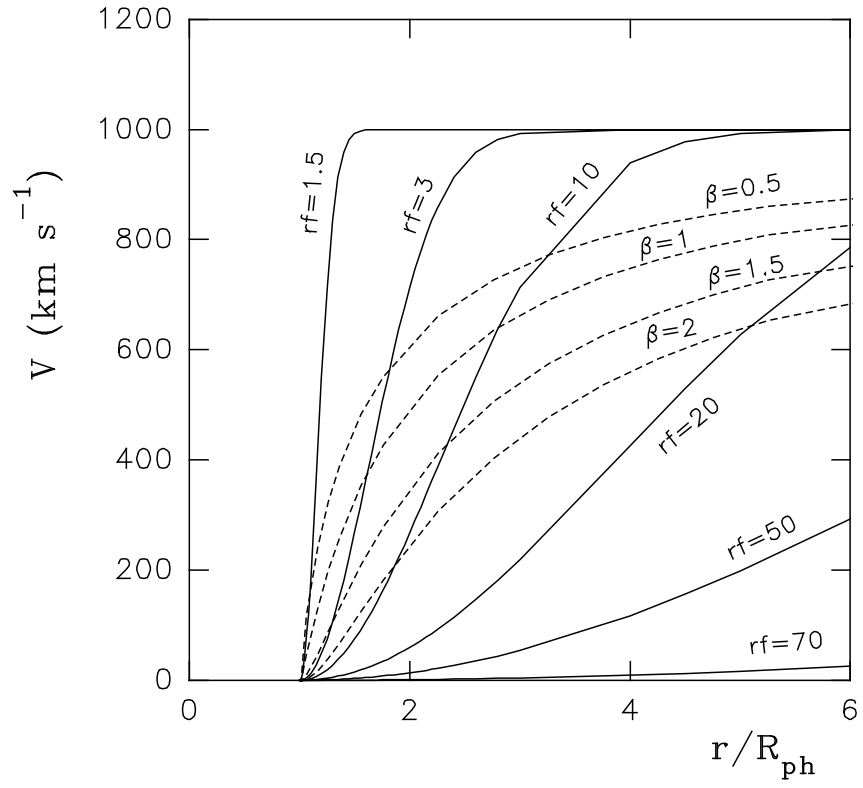

FIG. 1.-Comparison between the $\beta$ law (dotted line) and the law given by eq. (2) (solid line) for $V_{\infty}=1000 \mathrm{~km} \mathrm{~s}^{-1}$ and different values of $\beta$ and $r_{f}$.

the upper layers,

$$
V(r)=V_{0}+V_{\infty}\left(1-e^{-\alpha(r-1)^{2}}\right),
$$

where $\alpha=5 /\left(r_{f}-1\right)^{2}$. Equation (2) has two free parameters: $V_{\infty}$, the terminal velocity, and $\alpha$, an indicator of the degree of steepness of the wind defined through $r_{f}$, the radius at which the velocity approaches $V_{\infty}$.

As shown in Figure 1, in the innermost layers above the photosphere, the velocity law described by equation (2) has a different convexity and, as a consequence, the velocity gradient increases more smoothly than the one corresponding to the $\beta$ law; the maximum velocity can occur closer to the central star than in the case of the $\beta$ law.

Of stars display He II $\lambda \lambda 1640$ and 4686 in the velocity range between 0 and $600 \mathrm{~km} \mathrm{~s}^{-1}$ and superionized lines (Si IV, C Iv, etc.) between 0 and $2000 \mathrm{~km} \mathrm{~s}^{-1}$ (Grady et al. 1983). In order to represent the velocity reached in outermost parts of the He II line formation region, we adopt values for $V_{\infty}$ between 300 and $1200 \mathrm{~km} \mathrm{~s}^{-1}$. We see below that $V_{\infty}$ is not an important parameter for modeling He II lines, in the sense that it does not modify substantially the density law (see Fig. 2c), and the computed profiles present a similar appearance $(\S 4.1 .2)$.

The initial velocity, $V_{0}$, and the mass-loss rate are computed by means of the continuity equations with the iterative procedure described by Catala \& Kunasz (1987), adopting the photospheric density given by Kurucz (1979) models and the value $\tau_{c}=2 / 3$ for the continuum optical depth at the stellar (photospheric) radius. Thus, the massloss rate is computed consistently with the velocity law and the main contribution comes from the bottom of the wind. The values of the mass loss range between $1.7 \times 10^{-5} M_{\odot}$ $\mathrm{yr}^{-1}$, for the models with the highest velocity gradients, and $7.0 \times 10^{-10} M_{\odot} \mathrm{yr}^{-1}$, for those with the lowest velocity gradients. The mass density structure above $\tau_{c}=2 / 3$ is calculated by means of the continuity equation. The runs of densities corresponding to some of the models described in
Table 1 are illustrated in Figure 2. Figures $2 a$ and $2 b$ show the density laws obtained from equation (2) and the $\beta$ law, respectively, with $r_{f}=1.5,3$, and 70 and $\beta=0.8,1$, and 1.5. Here, we can observe that the larger the value of $r_{f}$ in equation (2), the denser the wind in the inner part of the atmospheres. Figure $2 c$ illustrates that runs of the densities corresponding to models with different terminal velocities are similar when the mass-loss rate is calculated consistently with the velocity law.

Below the layer corresponding to $\tau_{c}=2 / 3$, all the computed models have a photospheric structure in both radiative and in quasi-hydrostatic equilibrium. This region contributes to the continuous radiation, but it is optically thick in the line radiation. In order to represent these conditions, we have adopted the density and temperature stratification from the Kurucz (1979) model for a given $T_{\text {eff }}$ and $\log g$. We consider photospheres with effective temperatures ranging from 25,000 to $45,000 \mathrm{~K}$ and values of $\log g$ between 3.0 and $5.0 \mathrm{dex}$. The density distributions for different values of $\log g$ is presented in Figure $2 d$.

In the expanding region lying above the photosphere, we consider three different temperature stratifications, in order to represent cases of departure from radiative equilibrium. These layers are modeled either with a decreasing temperature law or with a moderate rise in the temperature distribution (where the maximum temperature value is a few times the effective temperature).

The basic temperature structures we considered are

1. A hot intermediate region (HIR). The layers above the photosphere increase in temperature until they reach a maximum and then drop down to a constant value. In the extended outer isothermal region the density is very low and the velocity reaches the terminal value. The temperature law is represented by the analytical expression proposed by Catala, Kunasz, \& Praderie (1984). This law includes the free parameters $\Delta_{1}$ and $\Delta_{2}$, which determine, respectively, the position and the width of the hot region. Here $T_{\max }$ is the maximum temperature value, and $T_{0}$ is the temperature of the isothermal outer envelope, which is assumed to be lower than the effective temperature.

2. A hot external region (HER). These models consider that the temperature increases with radius until it reaches a constant value, just a few times the effective temperature. The parameters describing these models are the temperature of the external region, $T_{0}$, and the radius, $\Delta_{1}$, at which $T_{0}$ is reached.

3. A cool external region (CER). All the layers above the photosphere have a constant temperature, $T_{0}$, lower than $T_{\text {eff. }}$

Figure 3 shows the three temperature distributions that correspond to cases 1,2 , and 3 , respectively.

It is important to note that for HER and HIR cases the rise in the temperature is around $10,000 \mathrm{~K}$ over the effective temperature of the star. This moderate heating might be mechanically produced by photospheric turbulent motion or acoustic wave dissipation.

In Table 1, we present the parameters of the most relevant models.

\section{RADIATIVE TRANSFER AND ATOMIC MODEL}

We solve rigorously the continuum and line transfer equations by Feautrier's method in the comoving frame 

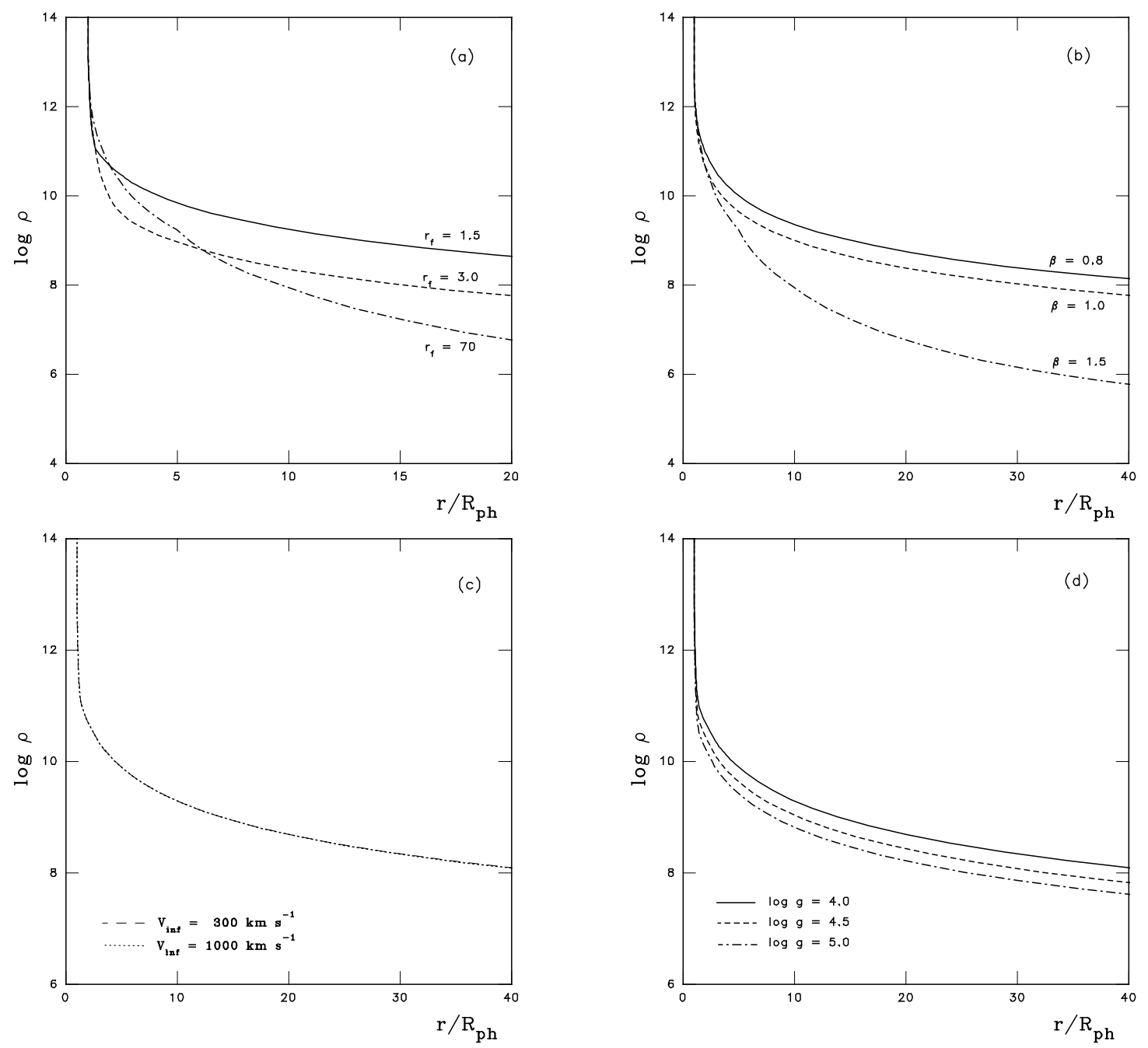

FIG. 2.- (a) Number density distributions corresponding to the velocity law described by eq. (2) for three velocity gradients $\left(r_{f}=1.5,3\right.$, and 70). (b) Number density distributions obtained with the $\beta$ law for $\beta=0.8,1$, and 1.5. (c) Two similar number density distributions derived with different terminal velocities and their corresponding mass-loss rates (eq. [2] for $v_{\infty}=300$ and $1000 \mathrm{~km} \mathrm{~s}^{-1}$ ). (d) Number density distributions for models with different log $g$.

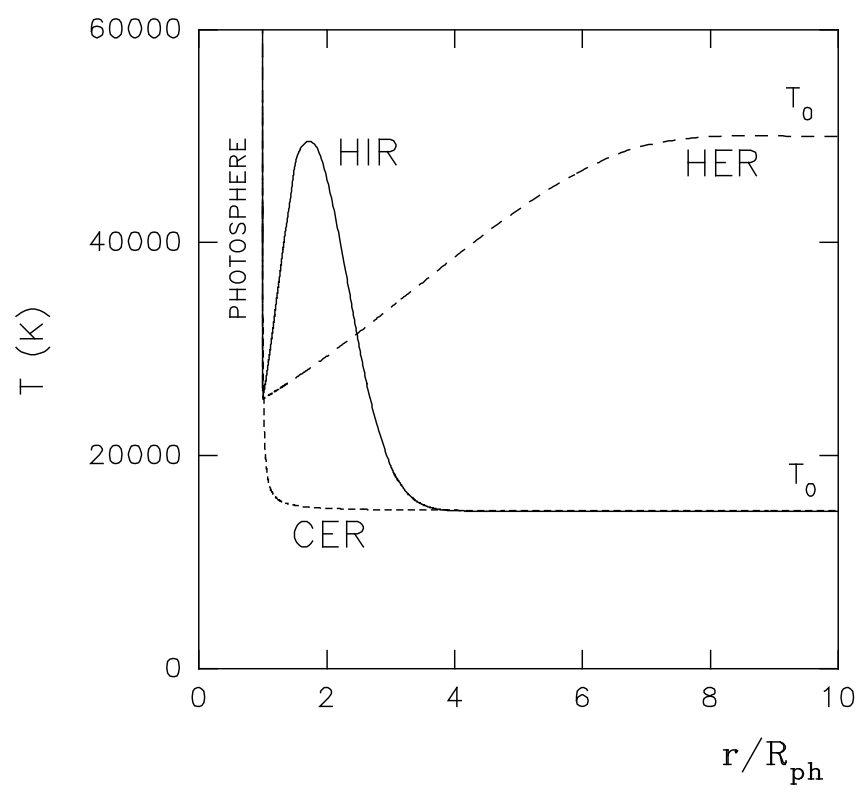

FIG. 3.-Temperature distributions representing a CER, an HER, and an HIR. for a spherically symmetric medium. A description of the method can be found in Mihalas \& Kunasz (1978) and references therein. The line transfer equation is solved consistently with the rate equations for multilevel atoms. We use the equivalent two-level atom approximation (ETLA) in order to obtain the source function. The lines are broadened by Doppler random motions with speeds up to $40 \mathrm{~km} \mathrm{~s}^{-1}$, and a complete redistribution is assumed. We do not consider the overlapping with $\mathrm{H}$ lines, since it does not affect the $\lambda 4686$ emission (Herrero 1987).

The ionized helium atom is a hydrogenic one, and in this study it is modeled by considering 10 bound levels and a continuum. This configuration allows us to calculate 45 explicit lines and 10 bound-free transitions. Recombinations to $\mathrm{He} \mathrm{I}$ are always considered to take place to the ground level. The He II cross sections for photoionization are given by Catala \& Kunasz (1987). The free-free cross section corresponds to the ones for hydrogen, but scaled up by a factor $Z^{2}$. Ionization and excitation collision rates were taken from Klein \& Castor (1978). 
TABLE 1

Selected Models of the Computed He il Lines

\begin{tabular}{|c|c|c|c|c|c|c|c|c|}
\hline Model & $\begin{array}{l}T_{\text {eff }} \\
(\mathrm{K})\end{array}$ & $\log g$ & Velocity Law & $\begin{array}{c}v_{\infty} \\
\left(\mathrm{km} \mathrm{s}^{-1}\right)\end{array}$ & $r_{f}$ or $\beta$ & $\begin{array}{c}\Delta_{1} \\
\left(R_{\mathrm{ph}}\right)\end{array}$ & $\begin{array}{c}T_{0} \\
(\mathrm{~K})\end{array}$ & $\begin{array}{c}\dot{M} \\
\left(M_{\odot} \mathrm{yr}^{-1}\right)\end{array}$ \\
\hline HIR-1 ......... & 25,000 & 3.0 & Eq. (2) & 300 & 1.5 & 2.00 & 8,000 & $1.85 \times 10^{-6}$ \\
\hline HIR-2 .......... & 25,000 & 3.0 & Eq. (2) & 300 & 70 & 2.00 & 8,000 & $7.29 \times 10^{-9}$ \\
\hline HIR-3 ......... & 25,000 & 3.5 & Eq. (2) & 300 & 1.5 & 0.33 & 8,000 & $9.08 \times 10^{-7}$ \\
\hline HIR-4 ......... & 25,000 & 3.5 & Eq. (2) & 300 & 1.5 & 2.00 & 8,000 & $8.95 \times 10^{-7}$ \\
\hline HIR-5 ......... & 25,000 & 3.5 & Eq. (2) & 300 & 3.0 & 0.33 & 8,000 & $1.18 \times 10^{-7}$ \\
\hline HIR-6 .......... & 25,000 & 3.5 & Eq. (2) & 300 & 3.0 & 1.00 & 8,000 & $1.17 \times 10^{-7}$ \\
\hline HIR-7 .......... & 25,000 & 3.5 & Eq. (2) & 300 & 3.0 & 3.00 & 8,000 & $1.16 \times 10^{-7}$ \\
\hline HIR-8 ......... & 25,000 & 3.5 & Eq. (2) & 300 & 3.0 & 5.00 & 8,000 & $1.16 \times 10^{-7}$ \\
\hline HIR-9 ......... & 25,000 & 3.5 & Eq. (2) & 300 & 10 & 0.33 & 8,000 & $2.23 \times 10^{-8}$ \\
\hline HIR-10 ....... & 25,000 & 3.5 & Eq. (2) & 300 & 10 & 2.00 & 8,000 & $2.21 \times 10^{-8}$ \\
\hline HIR-11 ....... & 25,000 & 3.5 & Eq. (2) & 300 & 70 & 0.33 & 8,000 & $3.71 \times 10^{-9}$ \\
\hline HIR-12 ....... & 25,000 & 3.5 & Eq. (2) & 300 & 70 & 2.00 & 8,000 & $3.65 \times 10^{-9}$ \\
\hline HIR-13 ....... & 25,000 & 4.0 & Eq. (2) & 300 & 1.5 & 2.00 & 8,000 & $4.89 \times 10^{-7}$ \\
\hline HIR-14 ....... & 25,000 & 4.0 & Eq. (2) & 300 & 70 & 2.00 & 8,000 & $2.03 \times 10^{-9}$ \\
\hline HIR-15 ....... & 25,000 & 4.5 & Eq. (2) & 300 & 70 & 2.00 & 8,000 & $1.15 \times 10^{-9}$ \\
\hline HIR-16 ........ & 25,000 & 5.0 & Eq. (2) & 300 & 1.5 & 2.00 & 8,000 & $1.53 \times 10^{-7}$ \\
\hline HIR-17 ....... & 25,000 & 5.0 & Eq. (2) & 300 & 70 & 2.00 & 8,000 & $6.52 \times 10^{-10}$ \\
\hline HIR-18 ....... & 40,000 & 4.0 & Eq. (2) & 300 & 1.5 & 2.00 & 15,000 & $2.53 \times 10^{-6}$ \\
\hline HIR-19 ....... & 40,000 & 4.0 & Eq. (2) & 300 & 1.5 & 2.00 & 25,000 & $2.52 \times 10^{-6}$ \\
\hline HIR-20 ....... & 40,000 & 4.0 & Eq. (2) & 300 & 3.0 & 0.33 & 15,000 & $3.33 \times 10^{-7}$ \\
\hline HIR-21 ....... & 40,000 & 4.0 & Eq. (2) & 300 & 70 & 0.33 & 15,000 & $9.82 \times 10^{-9}$ \\
\hline HIR-22 ....... & 40,000 & 4.0 & Eq. (2) & 300 & 70 & 3.0 & 15,000 & $9.73 \times 10^{-8}$ \\
\hline HIR-23 ....... & 40,000 & 4.0 & Eq. (2) & 1000 & 1.5 & 2.00 & 13,000 & $8.40 \times 10^{-6}$ \\
\hline HIR-24 ....... & 40,000 & 4.5 & Eq. (2) & 300 & 3.0 & 0.33 & 15,000 & $1.78 \times 10^{-7}$ \\
\hline HIR-25 ....... & 40,000 & 4.5 & Eq. (2) & 300 & 1.5 & 2.00 & 15,000 & $1.38 \times 10^{-6}$ \\
\hline HIR-26 ....... & 40,000 & 4.5 & Eq. (2) & 300 & 70 & 0.33 & 15,000 & $5.57 \times 10^{-9}$ \\
\hline HIR-27 ........ & 40,000 & 4.5 & Eq. (2) & 1000 & 1.5 & 2.00 & 13,000 & $4.61 \times 10^{-6}$ \\
\hline HIR-28 ....... & 40,000 & 5.0 & Eq. (2) & 300 & 1.5 & 2.00 & 15,000 & $8.46 \times 10^{-7}$ \\
\hline HIR-29 ....... & 40,000 & 5.0 & Eq. (2) & 300 & 3.0 & 0.33 & 15,000 & $1.09 \times 10^{-7}$ \\
\hline HIR-30 ....... & 40,000 & 5.0 & Eq. (2) & 300 & 70 & 0.33 & 15,000 & $3.48 \times 10^{-9}$ \\
\hline HIR-31 ....... & 40,000 & 5.0 & Eq. (2) & 1000 & 1.5 & 2.00 & 13,000 & $2.83 \times 10^{-6}$ \\
\hline HER-1 ......... & 25,000 & 3.0 & Eq. (2) & 300 & 70 & 7.00 & 30,000 & $7.40 \times 10^{-9}$ \\
\hline HER-2 ......... & 25,000 & 4.0 & Eq. (2) & 300 & 70 & 7.00 & 30,000 & $2.05 \times 10^{-9}$ \\
\hline HER-3 ........ & 25,000 & 4.0 & Eq. (2) & 300 & 70 & 8.00 & 30,000 & $2.05 \times 10^{-6}$ \\
\hline CER-1 ........ & 25,000 & 3.5 & Eq. (2) & 300 & 1 & $\ldots$ & 8,000 & $1.03 \times 10^{-6}$ \\
\hline CER-2 ........ & 25,000 & 3.5 & Eq. (2) & 300 & 1 & $\ldots$ & 8,000 & $1.03 \times 10^{-6}$ \\
\hline CER-3 ........ & 40,000 & 4.0 & $\beta$ & 1200 & 1 & $\ldots$ & 15,000 & $7.07 \times 10^{-6}$ \\
\hline CER-4 ........ & 40,000 & 4.0 & $\beta$ & 1200 & 1 & $\ldots$ & 20,000 & $4.99 \times 10^{-6}$ \\
\hline CER-5 ........ & 40,000 & 4.0 & $\beta$ & 1200 & 1 & $\ldots$ & 22,000 & $7.17 \times 10^{-6}$ \\
\hline CER-6 ......... & 40,000 & 4.0 & $\beta$ & 1200 & 1 & $\ldots$ & 23,500 & $7.19 \times 10^{-6}$ \\
\hline CER-7 .............. & 40,000 & 4.0 & $\beta$ & 1200 & 1 & $\ldots$ & 25,000 & $7.21 \times 10^{-6}$ \\
\hline CER-8 ........ & 40,000 & 4.0 & $\beta$ & 1200 & 1 & $\ldots$ & 26,000 & $7.21 \times 10^{-6}$ \\
\hline CER-9 ........ & 40,000 & 4.0 & $\beta$ & 1200 & 0.8 & $\ldots$ & 32,000 & $1.69 \times 10^{-5}$ \\
\hline CER-10....... & 40,000 & 4.5 & $\beta$ & 1200 & 0.8 & $\ldots$ & 32,000 & $1.12 \times 10^{-5}$ \\
\hline CER-11....... & 40,000 & 5.0 & $\beta$ & 1200 & 0.8 & $\ldots$ & 32,000 & $7.84 \times 10^{-6}$ \\
\hline
\end{tabular}

\section{RESULTS}

The models we analyze provide us with a description of the radiation field in the He II $\lambda \lambda 1640,3203$, and 4686 lines that emerge from a spherically expanding atmosphere. The results are presented for different values of $\log g$ and several wind velocity structures, which correspond to each temperature distribution presented in $\S 2$. The computed grid of models spans from 25,000 to $45,000 \mathrm{~K}$, in steps of $5000 \mathrm{~K}$; however, in order to describe the influence of the physical properties on the line profiles, we have selected from the whole set of models only those with $T_{\text {eff }}=25,000 \mathrm{~K}$, as representative of the global behavior in early B stars $\left(T_{\text {eff }}<30,000 \mathrm{~K}\right)$, or $T_{\text {eff }}=40,000 \mathrm{~K}$, for the intermediate O types $\left(T_{\text {eff }}>35,000 \mathrm{~K}\right)$.

\subsection{Hot Intermediate Region (HIR)}

4.1.1. Influence of Varying the Effective Gravity

In this section, we describe the response of He II line profiles to variations in the surface gravity. We found that all the computed transitions are sensitive to gravity and that, in some cases, the emission line intensity and the luminosity class are strongly correlated.

For a star with $T_{\text {eff }}=25,000 \mathrm{~K}$, Figure 4 shows the intensity variations of the normalized $\mathrm{P}$ Cygni line profile of $\mathrm{He}$ II $\lambda \lambda 1640$ and 4686 with effective gravity. A decrease in gravity yields an intensity enhancement of the emission and an increase of the equivalent width of both absorption and emission components (models HIR-1, HIR-4, HIR-13, and HIR-16). In Figure 5, we display 

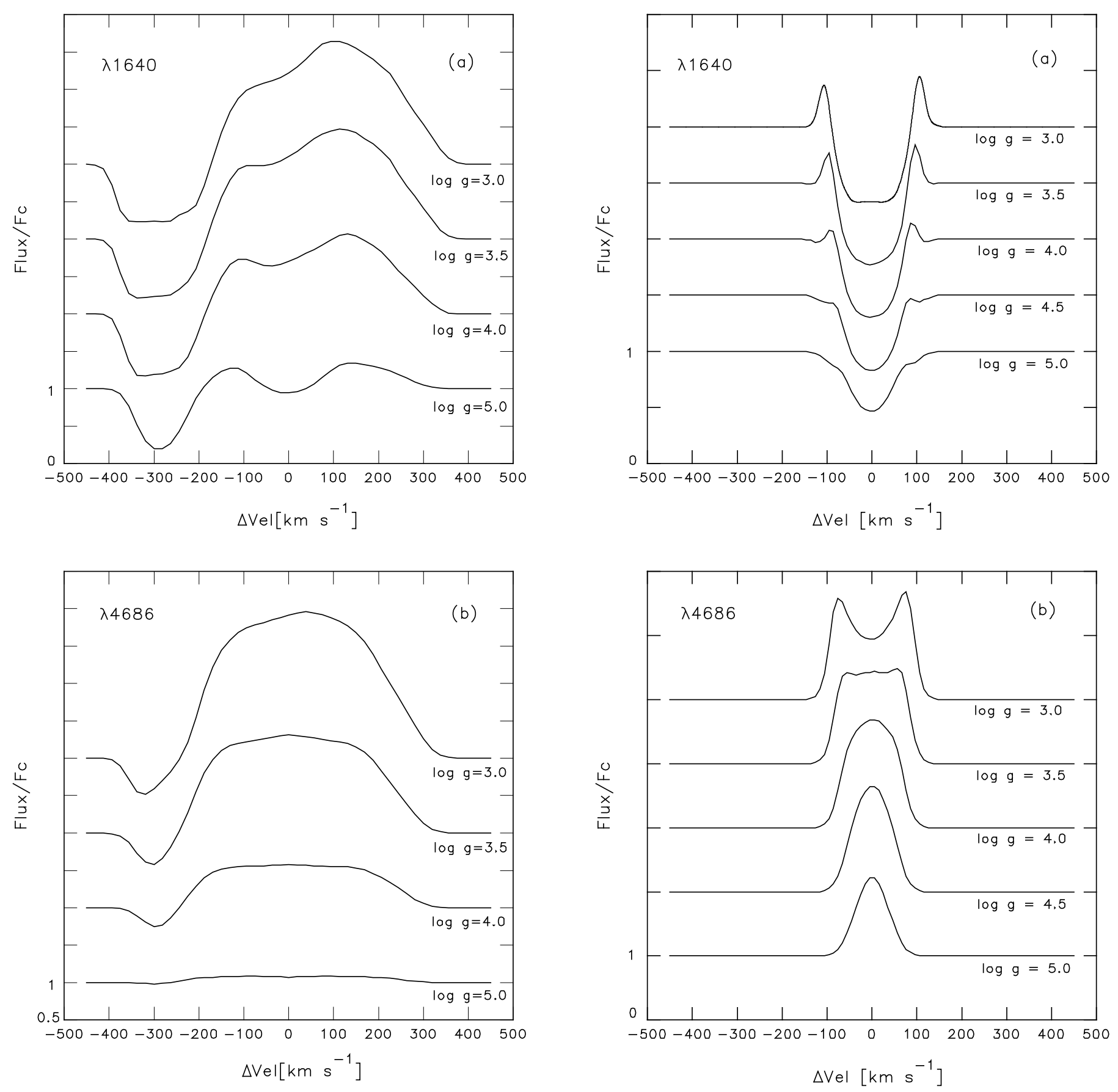

FIG. 4.-Changes in the computed He II lines due to variations in the surface gravity for (a) $\lambda 1640$ and $(b) \lambda 4686$. The models correspond to a star with $T_{\text {eff }}=25,000 \mathrm{~K}$, a hot temperature region at $\Delta_{1}=2$, and a fast wind with $r_{f}=1.5$ (models HIR-1, HIR-4, HIR-13, and HIR-16 in Table 1).

the resulting profiles for a less steepened wind (models HIR-2, HIR-12, HIR-14, HIR-15, and HIR-17). The line at $\lambda 1640$ appears in absorption for the largest gravity values and develops emission in the wings for the lowest values. The line at $\lambda 4686$ appears in emission and increases its intensity as lower values of $\log g$ are considered. For models with the lowest gravity values, a central reversal component forms.

For $T_{\text {eff }}=40,000 \mathrm{~K}$, the He II $\lambda 4686$ intensity variations with gravity are illustrated in Figure 6. As the gravity decreases, the absorptions turn into emissions: an absorp-

FIG. 5.- Same as Fig. 4, but models correspond to a star with $T_{\text {eff }}=25,000 \mathrm{~K}$, a hot temperature region at $\Delta_{1}=2$, and a slow expanding wind with $r_{f}=70$ (models HIR-2, HIR-12, HIR-14, HIR-15, and HIR-17 in Table 1).

tion profile evolves to a complex P Cygni profile (see Fig. $6 a$, models HIR-18, HIR-25, and HIR-28; and Fig. $6 b$, models HIR-23, HIR-27, and HIR-31), or to a single symmetric emission profile (Fig. 6c, models HIR-21, HIR-26, and HIR-30).

With respect to $\lambda 1640$, the same sequences of models (HIR-18, HIR-25, HIR-28 and HIR-23, HIR-27, HIR31) predict a P Cygni profile with an emission component that diminishes its intensity. Models HIR-21, HIR-26, and HIR-30 give a symmetric absorption profile for this line. 

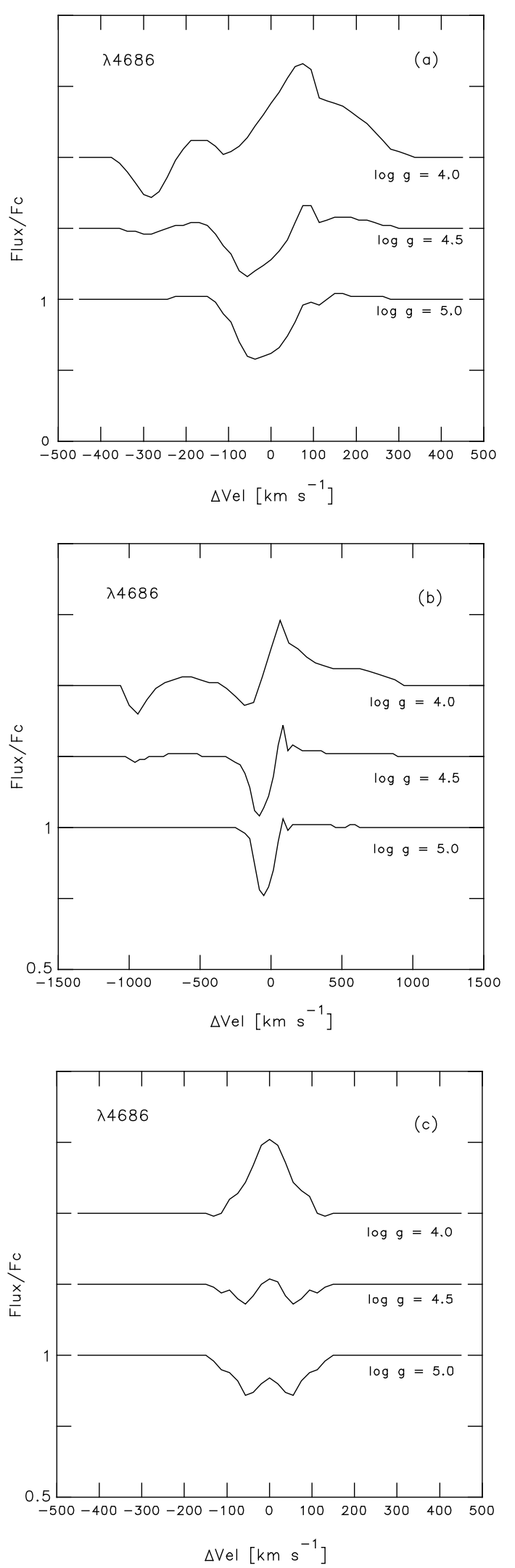

FIg. 6.- - He II $\lambda 4686$ line variations with gravity for a star with $T_{\text {eff }}=40,000 \mathrm{~K}$. (a) Profiles computed for a steepened gradient in the wind, $r_{f}=1.5, V_{\infty}=300 \mathrm{~km} \mathrm{~s}^{-1}$, and an HIR at $\Delta_{1}=2$ (models HIR-18, HIR-25, and HIR-28 in Table 1). (b) Same as (a), but for $V_{\infty}=1000 \mathrm{~km}$ $\mathrm{s}^{-1}$ (models HIR-23, HIR-27, and HIR-31). (c) Emergent flux profiles corresponding to a slow wind, $r_{f}=70, V_{\infty}=300 \mathrm{~km} \mathrm{~s}^{-1}$, and an HIR temperature law with $\Delta_{1}=0.33$ (models HIR-21, HIR-26, and HIR-30).
The effect of varying the gravity yields a different density distribution (see Fig. 2d). For models with the same velocity law, the lower the gravity, the higher the mass-loss rate and, therefore, the denser the wind. This leads to an enhancement of the intensity in the line.

The behavior shown in Figures $6 a-6 c$, where the absorption profile of He II $\lambda 4686$ turns into emission as the gravity decreases, is a result that occurs only for the highest effective temperatures, which correspond to O-type stars. This result is in accordance with the observational behavior of $\mathrm{He}$ II $\lambda 4686$, a line that is usually considered a luminosity indicator (Walborn 1971).

\subsubsection{Influence of Varying the Velocity Law}

We find it more appropriate to describe a line by means of the parameters of the velocity law than by using the density distribution, since the line profile function is mainly defined by the Doppler effect. A great variety of shapes of the line profiles are obtained by varying the radial velocity gradient at the base of the wind (close to the photosphere). In our models, this velocity gradient is controlled by the parameters $\beta$ or $r_{f}$ (see $\S 2$ ). Low values of $r_{f}$ (less than 3) and $\beta$ $(\leq 1)$ yield P Cygni profiles for all the computed transitions. As the velocity gradient next to the photosphere decreases (by increasing $r_{f}$ or $\beta$ ), the P Cygni structure of the profile vanishes and $\mathrm{He}$ II $\lambda 1640$ turns into a symmetrical absorption, while He II $\lambda 4686$ becomes a symmetrical emission. In Figure 7, we display the $\lambda \lambda 1640$ and 4686 line profile changes due to different velocity gradients at the base of the wind (models HIR-3, HIR-5, and HIR-11). Although the figure shows only synthetic line profiles computed for $T_{\text {eff }}=25,000 \mathrm{~K}$, this result holds over the whole effective temperature range being considered.

For intermediate $r_{f}$-values $\left(r_{f} \sim 3\right)$, the He II $\lambda 1640$ line can still display a P Cygni-like feature, while the other transitions present a symmetric profile. In some cases, $\lambda 1640$ exhibits a P Cygni profile with a second blueward absorption that marks the flow velocity at the outer layers. The relative intensity of the second absorption component is very sensitive to gravity and to the temperature law. The behavior with gravity is shown in Figure 8 for a sequence of models with $T_{\text {eff }}=40,000 \mathrm{~K}$. Details of the temperature law response of this component are commented on in $\S 4.1 .3$.

It is important to stress that the main parameter that governs the shape of the profile is $r_{f}$ or $\beta$; other parameters (e.g., the terminal velocity) give rise to intensity variations but keep the characteristic shape of the profile. A comparison between Figures $6 a$ and $6 b$ puts this fact in evidence. As $V_{\infty}$ increases, the $\mathrm{P}$ Cygni profile becomes wider, the absorption component moves toward the blue, and the emission component decreases in intensity and spreads into the wings. The same behavior was previously obtained by Hamann \& Schmutz (1987).

\subsubsection{Influence of Varying the Parameters of the High- Temperature Region}

The He II $\lambda \lambda 1640$ and 4686 lines present different responses to the variation of the parameters of the temperature law. The effects produced by the temperature should be added to those related to the velocity field and the gravity, already described in $\S \S 4.1 .1$ and 4.1.2. Of the temperature law parameters mentioned in $\S 2$, we set $\Delta_{2}=1.5 R_{\text {ph }}$ and $T_{\max }=40,000 \mathrm{~K}$ (for $T_{\text {eff }}=25,000 \mathrm{~K}$ ) or $50,000 \mathrm{~K}$ (for 

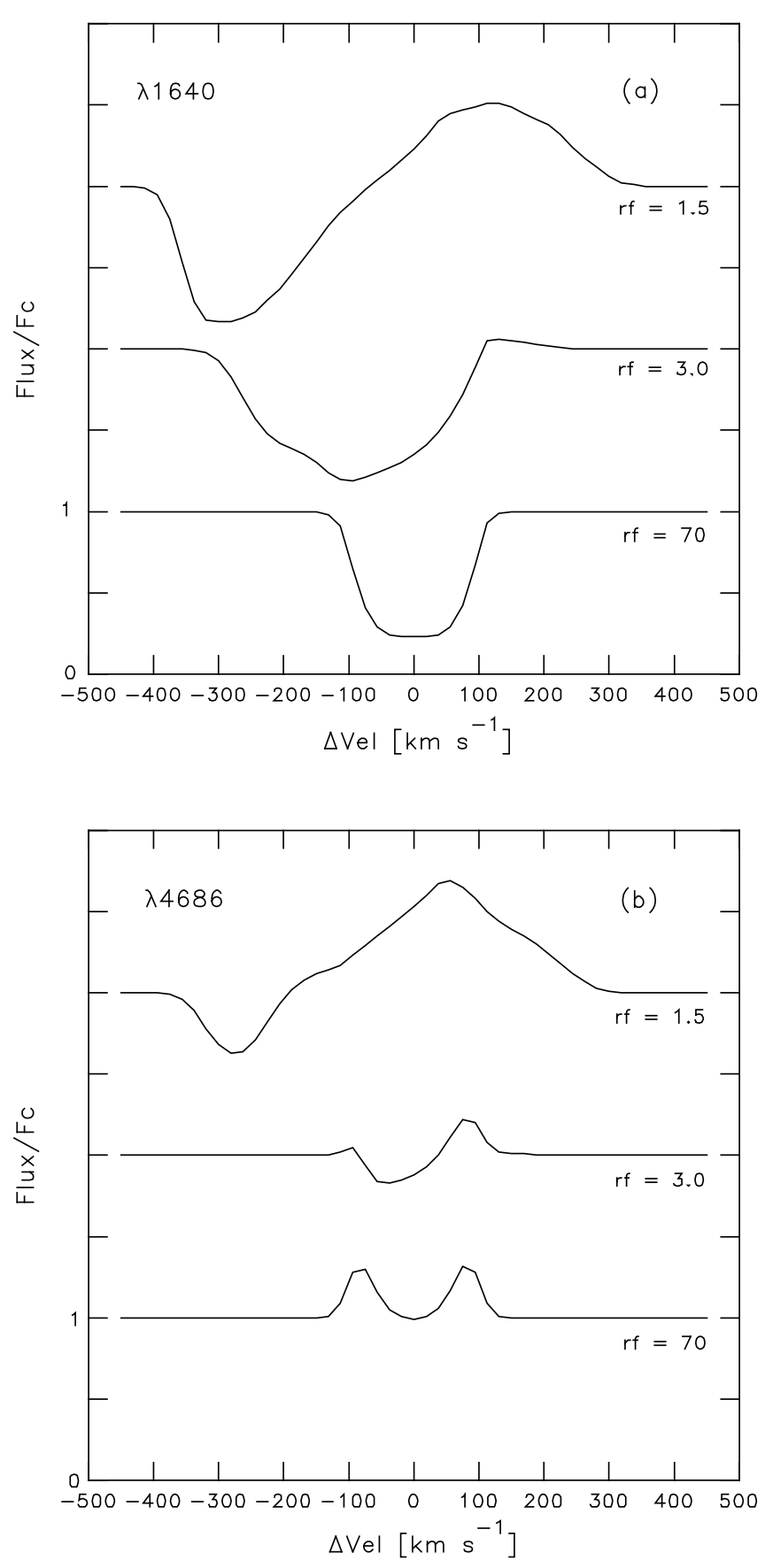

FIG. 7.- Response of He II lines to the velocity gradient parameter $r_{f}$ for (a) $\lambda 1640$ and (b) $\lambda 4686$. Models HIR-3, HIR-5, and HIR-11 correspond to $T_{\text {eff }}=25,000 \mathrm{~K}, \log g=3.5$, and $\Delta_{1}=0.33$.

$T_{\text {eff }}=40,000 \mathrm{~K}$ ), since the line profiles are less sensitive to these parameters.

We have varied the external temperature, $T_{0}$, between 0.3 and 0.6 of the effective temperature, fixing the remaining parameters. The resulting line profile keeps its shape, but the higher the values of $T_{0}$ the stronger the intensity of the emission and/or the absorption components of the lines. The line strength is a consequence of an increment on the occupational numbers of the He II upper levels due to the rise of $T_{0}$.

All the spectral types considered show almost the same response to variations in the location of the temperature

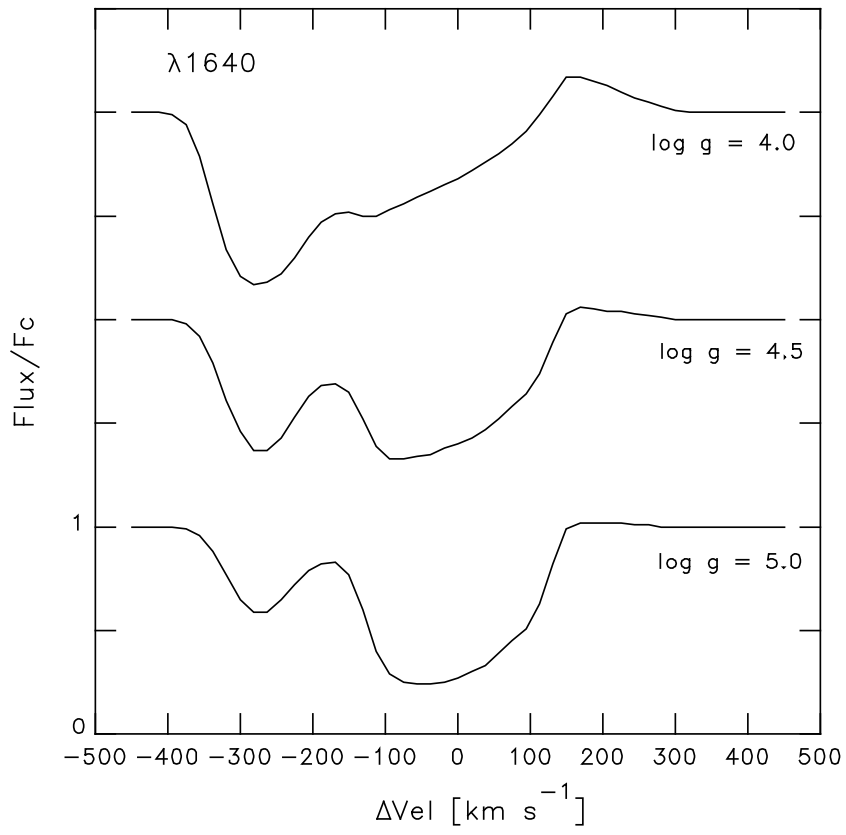

FIG. 8. - P Cygni profiles of $\lambda 1640$, with a second blueward absorption obtained with $r_{f}=3$ and a $T_{\text {eff }}=40,000 \mathrm{~K}$ (models HIR-20, HIR-24, and HIR-29 in Table 1). Both absorption components are sensitive to variations of $\log g$.

maximum. As the maximum temperature region moves outward from the photosphere (increasing parameter $\Delta_{1}$ ), the profiles undergo the following changes:

1. In He II P Cygni profiles with a second blueward absorption component, the second absorption becomes deeper, while the first absorption component is filled in by the red wing emission (this variation is illustrated for $\lambda 1640$ in Fig. 9).

2. In a P Cygni profile, there is an enhancement of the emission component, whereas the absorption is slightly broadened and shifted to the blue. The He II $\lambda 4686$ emission increases more slowly than the emission at $\lambda 1640$ (Fig. 10).

3 . For the cases of lower effective temperatures, the symmetric absorption profiles in $\lambda 1640$ can develop emissions in the wings and their strengths can increase progressively (Fig. 11). In the case of $T_{\text {eff }}=40,000 \mathrm{~K}$, the line remains in absorption, although its core is slightly filled by the emission.

4. The $\lambda 4686$ line, which presents a pure emission profile, enhances its intensity. In those cases in which a wing emission structure is evident in the absorption line, the profile becomes one of pure emission (Fig. 12).

In all these cases the emission increment is due to a greater emission volume. As $\Delta_{1}$ increases, the line formation region moves outward.

\subsection{Hot External Region (HER)}

The theoretical profiles obtained for the HER models, with the temperature increasing with the radius up to a value $T_{0}$, follow a behavior quite similar to those of profiles that are computed with the HIR models. The profiles are dominated by the velocity field, especially within the first stellar radius, which can produce either P Cygni profiles for high-velocity flows or symmetrical profiles for low-velocity 


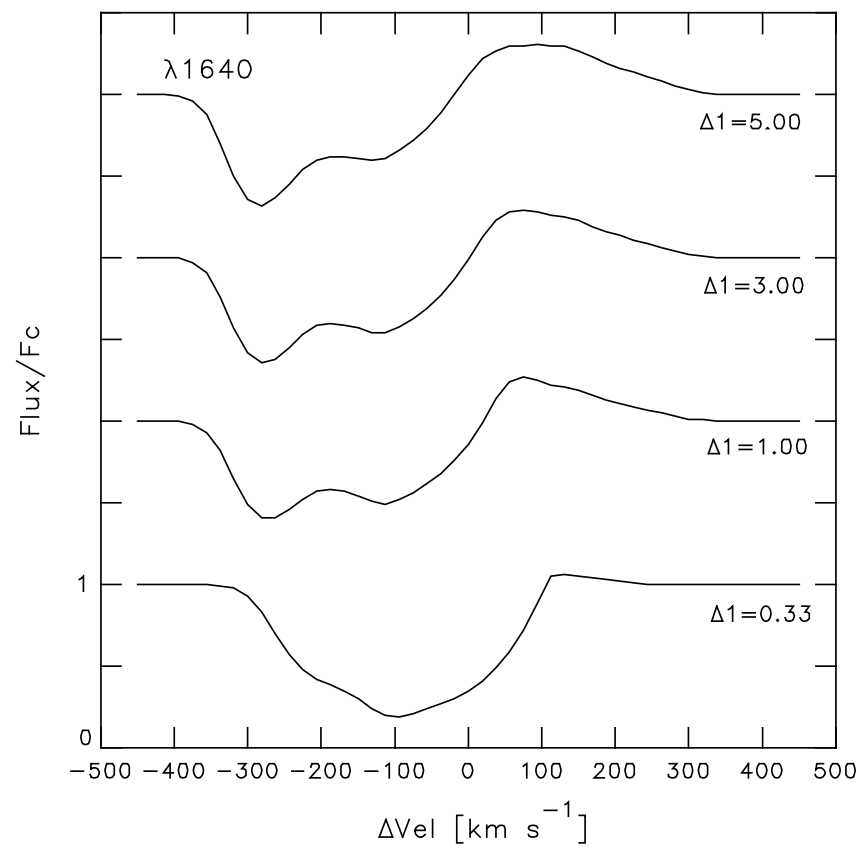

FIG. 9.-Absorption profile of $\lambda 1640$ becomes a P Cygni profile with two absorption components as the maximum temperature region moves outward (models HIR-5-HIR-8 in Table 1).

flows. We have run a large number of models, and we summarize here some significant general results. Only those models that are related to the figures have been included in Table 1.

With respect to the velocity law, for $T_{\text {eff-values between }}$ 25,000 and 40,000 $\mathrm{K}$ the He II lines show very strong emission in the P Cygni profile, when $r_{f}=1.5$ is considered. For greater values of $r_{f}$ (greater than 3), $\lambda 4686$ often displays a symmetric emission profile, even for the lowest-velocity regimes, in clear contrast with $\lambda 1640$, which is mainly in absorption.

Otherwise, all the lines are sensitive to gravity, in the same way as described for the HIR models: low gravities at the photospheric layers increase the emission rates. Sometimes, $\lambda 1640$ might develop emission in the wings for the lowest values of $\log g$. Line profile dependence with gravity is illustrated in Figure 13. The profiles are barely sensitive to $T_{0}$ and to the location of the HER, which induce only small changes in the global structure, and they keep the original line shape already determined by the velocity law.

\subsection{Cool External Region (CER)}

This section describes the results obtained with the isothermal envelope. Among the models we have considered, we found that, for low effective temperatures, the profiles are always in absorption, even for the steepest velocity regimes and the highest terminal velocities (Fig. 14). The strength of the line depends slightly upon the wind mass density, and it is quite insensitive to the temperature of the isothermal region, which was assumed to be lower than $0.8 T_{\text {eff }}$ (a typical temperature at the external border for a gray atmosphere).

In the case of models with high effective temperature, we note that the computed profiles resulting from the velocity
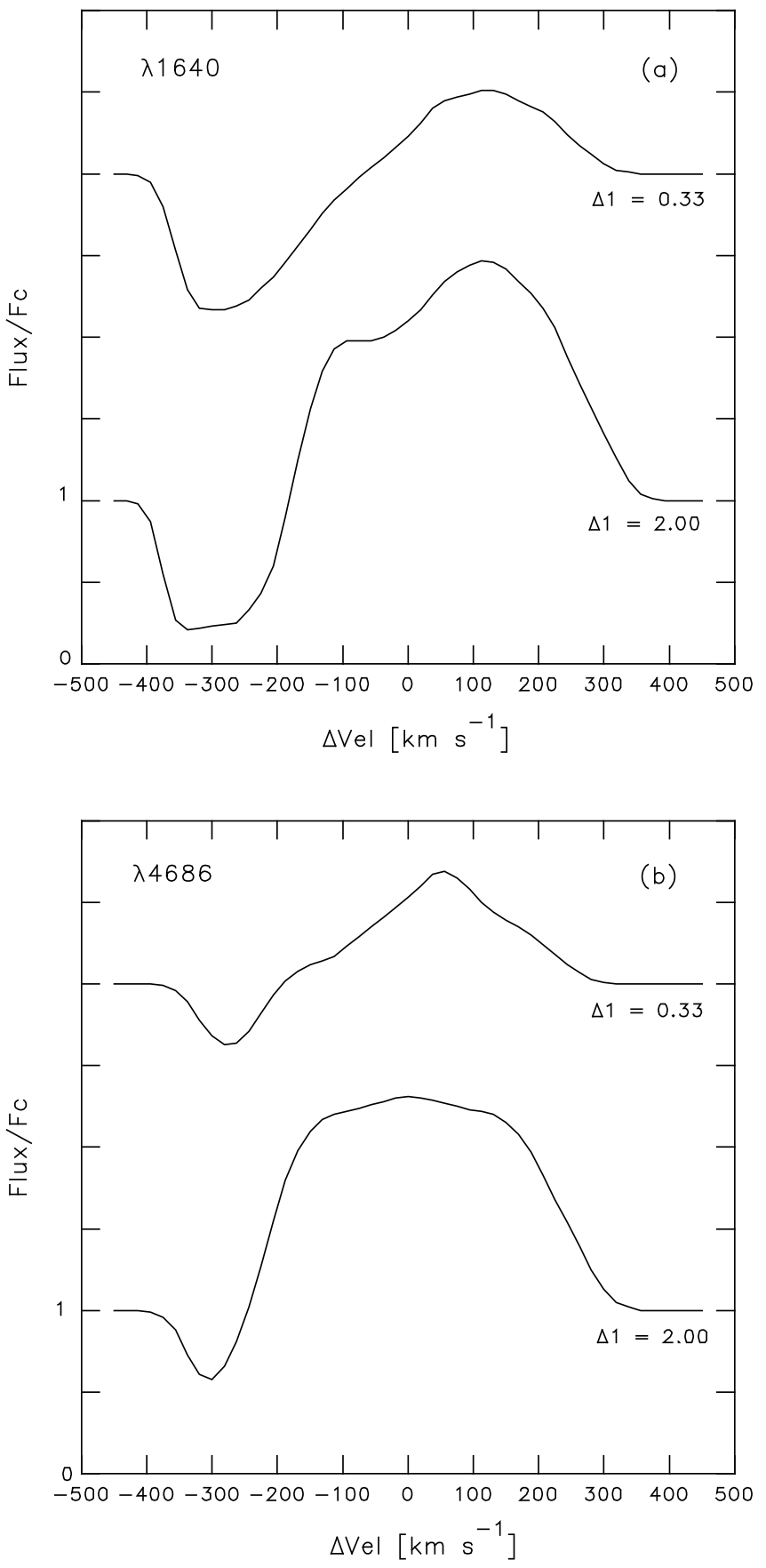

FIG. 10.-Variations of the P Cygni profiles when the maximum temperature region is shifted outward, for (a) $\lambda 1640$ and $(b) \lambda 4686$ (models HIR-3 and HIR-4 in Table 1).

laws given by equations (1) and (2) are quite similar, showing again that the profiles are quite insensitive to the proposed velocity laws. Models with a low-velocity regime, i.e., low terminal velocity or soft gradients at the base of the wind, produce only absorption profiles.

In clear contrast with the results obtained for low effective temperature, in the models with $T_{\text {eff }}=40,000 \mathrm{~K}$ and a high-velocity regime the main parameter that governs the appearance of the profiles is $T_{0}$, which can induce emission components. As $T_{0}$ increases from 15,000 to $22,000 \mathrm{~K}$, the $\lambda 1640$ line develops a second absorption 


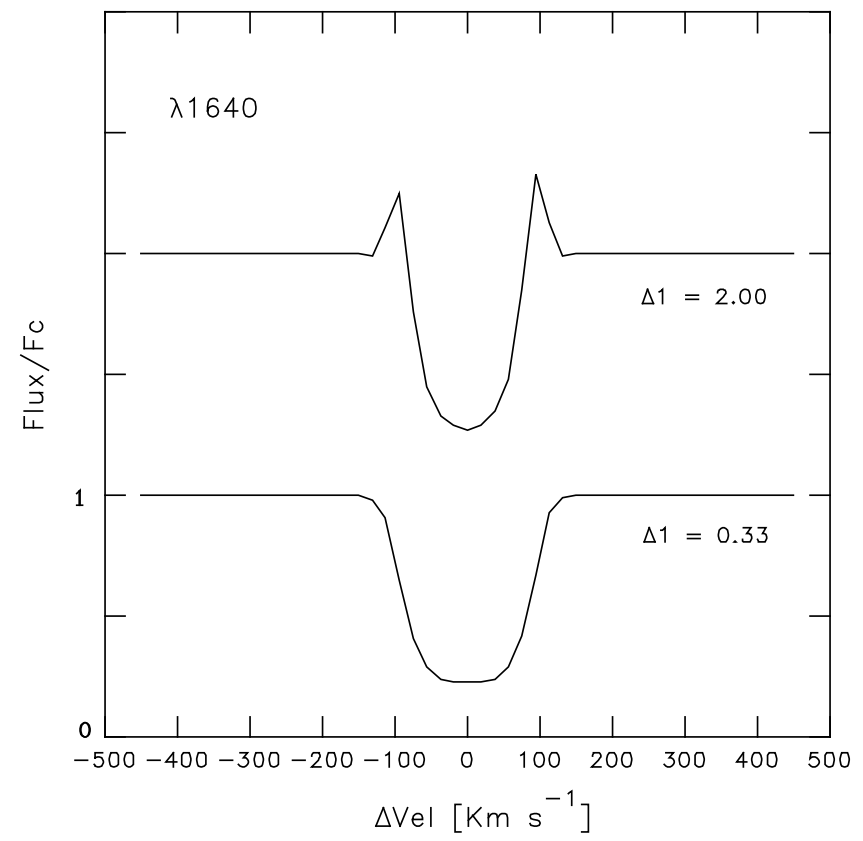

FIG. 11.-Symmetric $\lambda 1640$ in absorption develops emission in the wings when the location of the maximum temperature region is shifted outward. This result is expected for $T_{\text {eff }}=25,000 \mathrm{~K}$ (models HIR-11 and HIR-12 in Table 1).

component located close to the terminal velocity. Between 22,000 to $26,000 \mathrm{~K}$, the two absorption components merge into a single absorption, and an important emission in the red wing develops, yielding a P Cygni profile (Fig. 15a). For the same set of models, the $\lambda 4686$ line changes from an absorption to an emission profile as

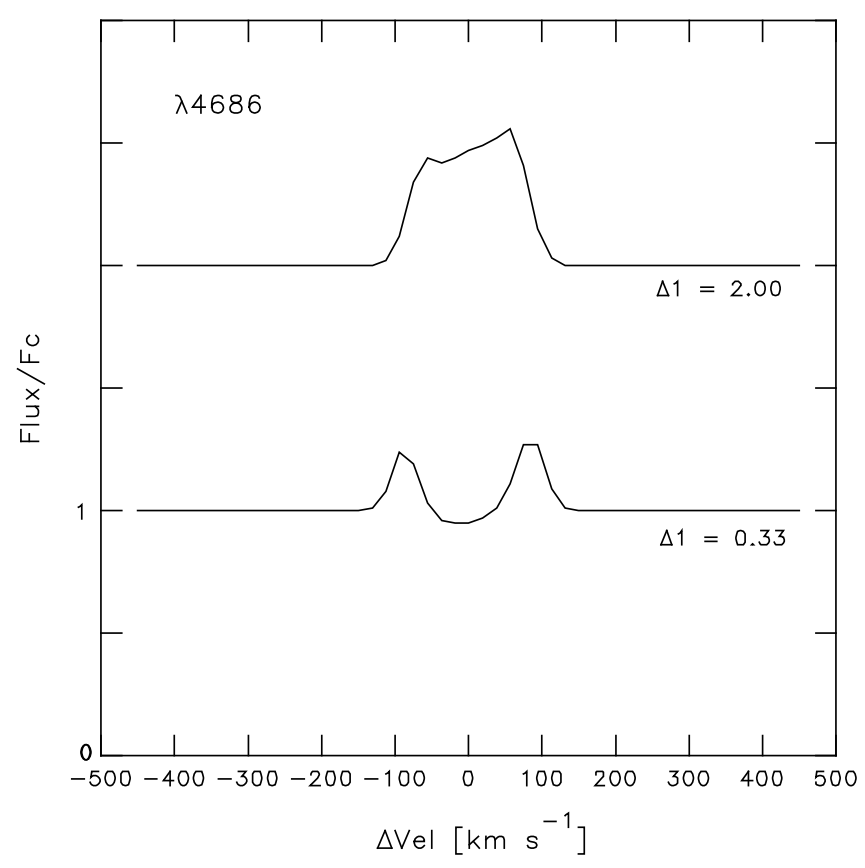

FIG. 12. - A $\lambda 4686$ emission profile becomes more intense when the maximum temperature region is moved outward (models HIR-9 and HIR-10 in Table 1).
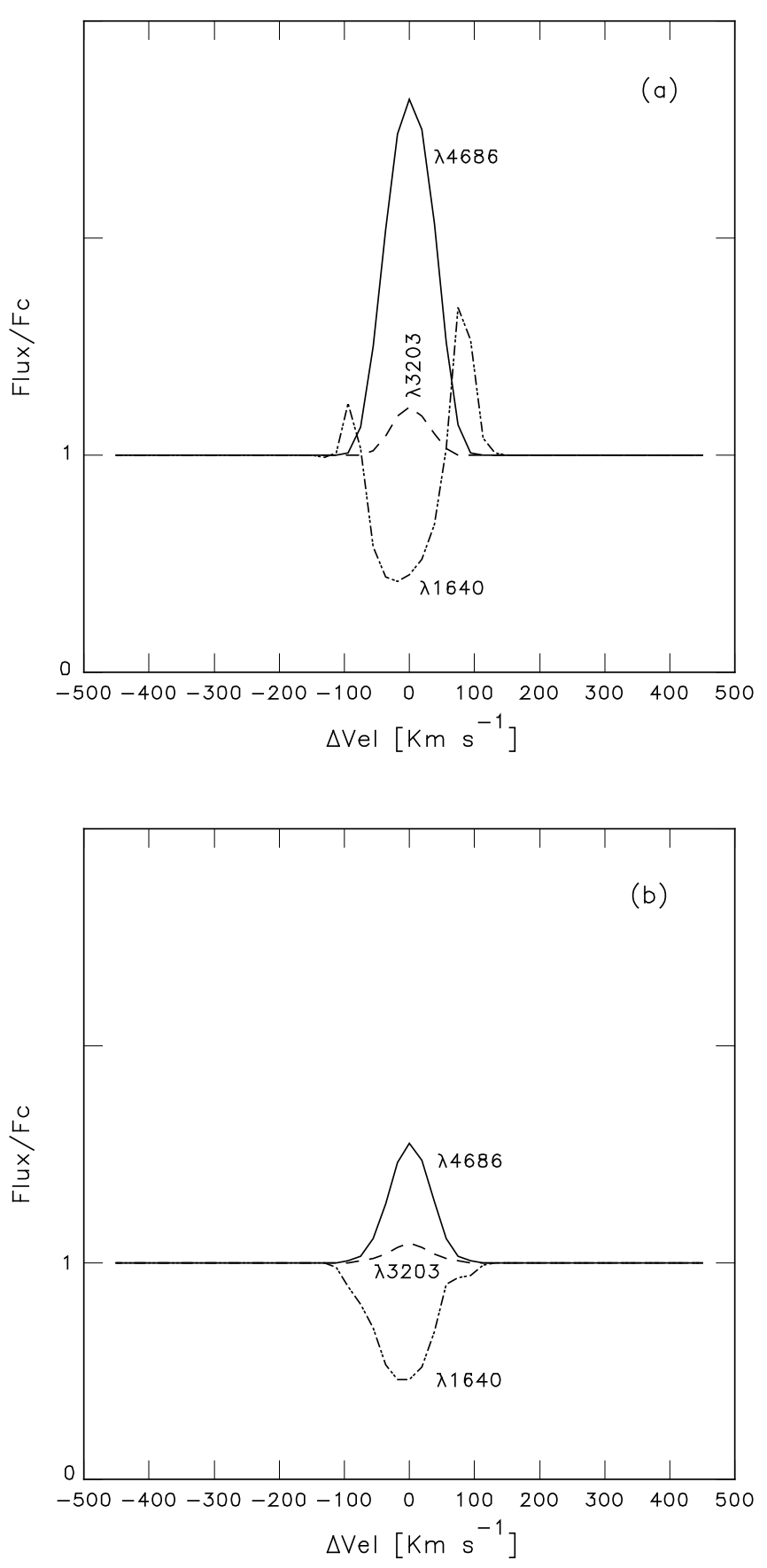

FIG. 13.-Synthetic line profiles of He II computed for ( $a$ ) model HER-1, with $\log g=3.0$ and a slow flow, $r_{f}=70$; and $(b)$ model HER-2, with $\log g=4.0$ and the same velocity regime.

the temperature $T_{0}$ increases (Fig. 15b). We find again that the occupational numbers of the upper levels increase with $T_{0}$.

A CER model with $T_{0}=0.8 T_{\text {eff }}$ and a $\beta$ law resembles a typical radiative equilibrium standard model. The line profile response to gravity variations for this particular case is plotted in Figure 16. As the effective gravity decreases, the mass density increases, yielding an enhancement of the intensity. We note that the line presents a P Cygni profile and does not change into a pure absorption, as in the case of HIR and HER models (already described in $\S \S 4.1 .1$ and 4.2). 


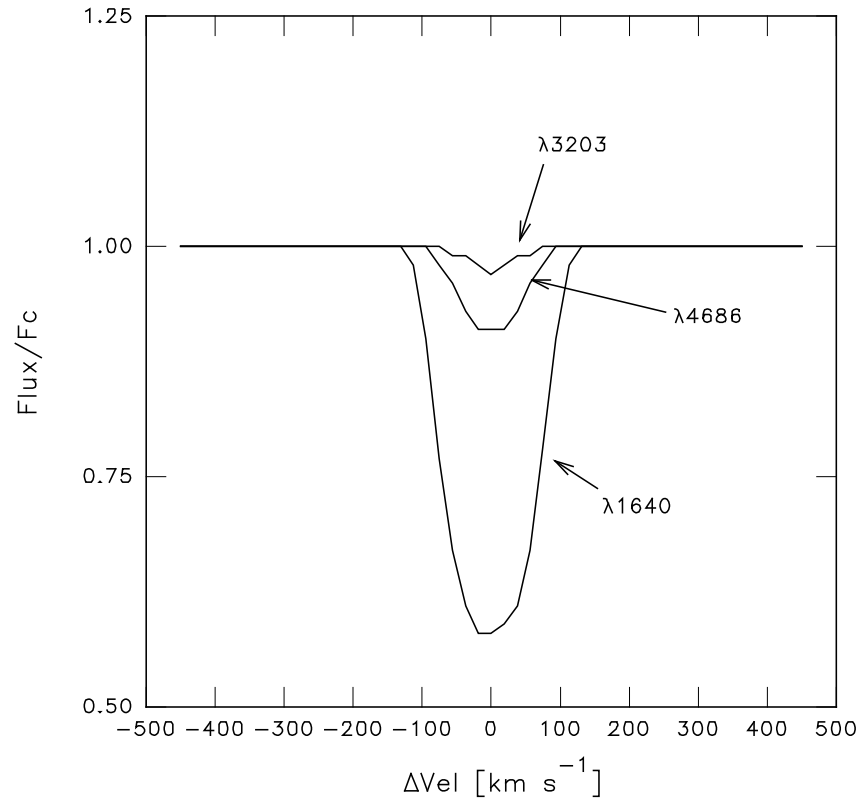

FIG. 14.-Normalized absorption-line profiles obtained for $T_{\text {eff }}=25,000 \mathrm{~K}$ and a high-velocity gradient at the base of the wind (model CER-1 in Table 1).

\section{DISCUSSION AND GENERAL REMARKS}

In this work we have derived He II emergent flux profiles, computed with a self-consistent solution of the continuum and NLTE line transfer equations for a spherical configuration. We have explored models with different temperature and velocity wind structure and their influence on the line profile.

We find that the conditions of the extended atmosphere determine the dominant characteristics in the appearance of the profiles (symmetrical or asymmetrical) and the presence of emission or absorption. The characteristic shape of the profiles is governed by the velocity gradient at the base of the wind whenever a positive temperature gradient occurs (i.e., in HER and HIR models). High velocity gradients yield P Cygni profiles, while low velocity gradients produce symmetric profiles.

From the analysis of the computed models, we verify that the presence of absorption and emission lines is a consequence of several different physical conditions. The emission rate in the lines increases for the models with $\operatorname{low} \log g$ and depends on the effective temperature, and on the temperature structure of the expanding region as well. For $T_{\text {eff }}=25,000 \mathrm{~K}$ (spectral type B), CER models predict absorption lines, while most of the HIR and the HER models tend to predict emission lines. CER models with $T_{\text {eff }}=40,000 \mathrm{~K}$ (spectral type O) display emission components in $\lambda 4686$ when $T_{0}$ exceeds $22,000 \mathrm{~K}$ (Fig. 15b). Higher values of $T_{0}$ can yield P Cygni profiles for $\lambda 1640$ and important emissions in $\lambda 4686$, as observed in Of stars. This is an expected result, since by increasing the temperature $T_{0}$ of an isothermal circumstellar region (CER model), the temperature structure of an HER-like model is reproduced. Therefore, we find again that the line profiles begin to develop emissions and that their shapes are affected by the velocity regime.
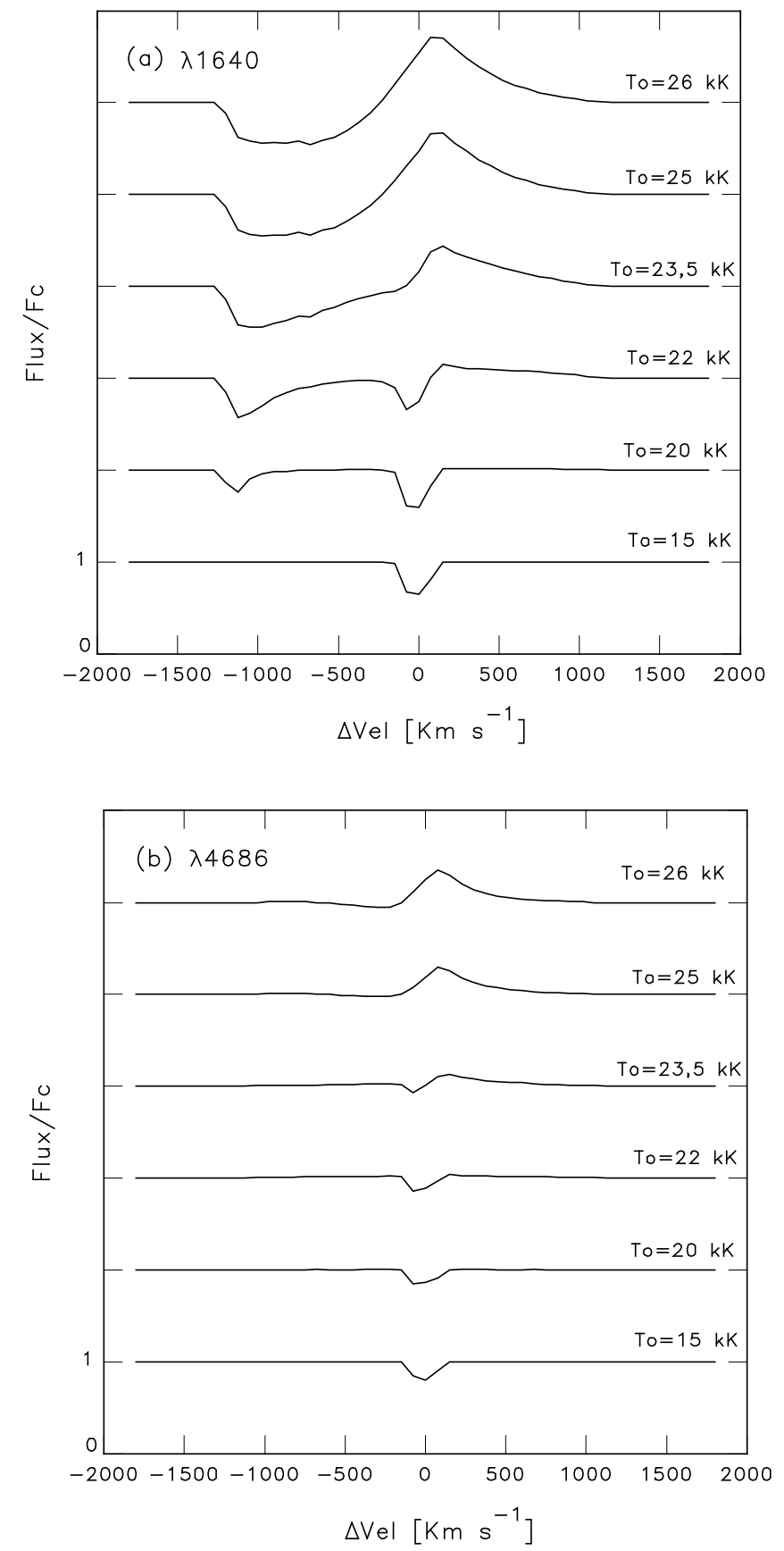

FIG. 15.- - He II line variations for (a) $\lambda 1640$ and $(b) \lambda 4686$, computed with CER models with $T_{\text {eff }}=40,000 \mathrm{~K}$ and $T_{0}$ ranging from 15,000 to 26,000 K (models CER-3-CER-8 in Table 1).

We conclude that a "warm," extended, and rapidly expanding atmosphere is a sufficient condition for obtaining emission in $\mathrm{He}$ II lines. The emission rate can also be increased by shifting the high-temperature region in an HIR structure toward larger radii.

For any given set of parameters $T_{\text {effi, }} \Delta_{1}$, and $r_{f}$, our models predict a line emission enhancement for low effective gravity. Only for HIR and HER models with high effective temperature $\left(T_{\text {eff }} \sim 40,000 \mathrm{~K}\right)$ can a line profile change from pure absorption to a $\mathrm{P}$ Cygni or a pure emission type when gravity decreases. Instead, among low effective temperature models, variations in the effective gravity do not 


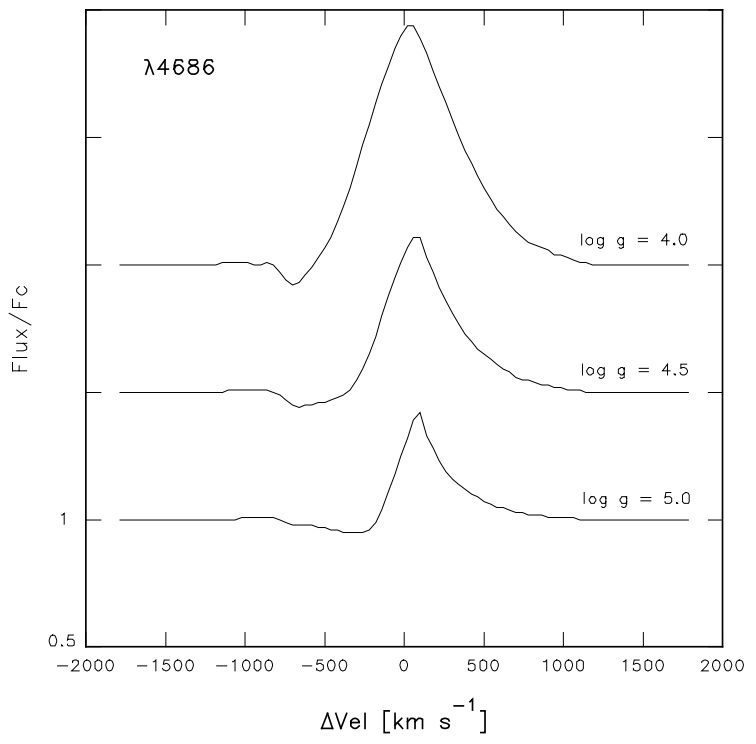

FIG. 16.-Line profile behavior with surface gravity for isothermal models with $T_{0}=0.8 T_{\text {eff }}$ (models CER-9-CER-11)

imply a significant modification in the shape of the profile, which remains quite the same.

It is important to stress that our models are limited to spherical configurations. It is difficult to predict the effects of differential rotation on a line profile, since its features would be affected by both a two-dimensional density stratification and Doppler effects due to the influence of the wind and the rotation. The computation of the radiation transfer equation in the comoving frame for a rotating stellar wind is still waiting to be undertaken. Up to now, the effect of rotation has only been explored with the Sobolev approximation (Mazzali 1990; Petrenz \& Puls 2000) but, unfortunately, this approximation is not adequate for the regions with low-velocity gradients proposed in this work. Even though line profiles presenting quasi-periodic variations might be explained by rotation, we have to keep in mind that nonperiodic line variability would be more consistent with changes in the mass-loss rate, in the wind ionization balance, or in the radial velocity field (Grady et al. 1983).

\section{COMPARISON WITH OBSERVED He II LINES}

A straightforward comparison of computed and observed line profiles would not be fully successful, since the velocity and temperature distributions in a particular star might not be the ones we considered in the line transfer calculation. We intended only to obtain a rough representation of the main features typically observed in the line profiles, in order to establish constraints on the general structure of the extended atmosphere.

In early B stars (and even in Be stars), $\lambda 4686$ is in absorption, if it appears at all. We find that almost all of the computed models with a temperature rise in the external regions produce emission components in this line. Exceptions are low- $T_{0}$ CER models and HIR models with a hot region very close to the photosphere, i.e., $\Delta_{1} \leq 1 R_{\text {ph }}$. Thus, we conclude that early B and Be stars cannot have a substantial rise in the temperature or that the high-temperature region must be located close to the photosphere. Instead, $\mathrm{B}[\mathrm{e}]$ stars can display important emissions in $\lambda 4686$ (Nota et al. 1996), indicating, perhaps, the presence of a high-temperature wind.

We do not find any physical condition that could produce a completely pure emission or P Cygni profile in $\lambda 1640$ with more than one absorption component, but as far as we know, these kinds of profiles have not been reported in OB stars. Conversely, synthetic He II $\lambda 4686$ in pure emission or complex P Cygni line profiles that look like those observed (Nota et al. 1996; Rauw et al. 2001) can often be obtained for a wide range of model parameters.

As $\mathrm{He}$ II $\lambda 3203$ is observed in absorption (Morrison 1975), it is also important to consider those parameters that induce this result. In Figure 17, we show the computed He II lines for four models that reproduce qualitatively the observed lines for different spectral types. The parameters for these models are given in Table 1. The first two models correspond to a star with $T_{\text {eff }}=40,000 \mathrm{~K}$ and $\log g=4.0$. The first one shows a strong P Cygni profile in $\lambda 1640$, a strong emission in $\lambda 4686$, and a shallow absorption in $\lambda 3203$ (Fig. 17a). The high velocity gradient of the wind is evident in the P Cygni shape of the profile. The second model shows symmetric profiles that correspond to a wind with a low velocity gradient (Fig. 17b); He II $\lambda \lambda 1640$ and 3203 are in absorption, while $\mathrm{He}$ II $\lambda 4686$ is in emission. The synthetic profiles shown in Figures $17 a$ and $17 b$ resemble those observed in Of-type stars. In Figure $17 b$, the intensity of the computed $\lambda 1640$ line is, in general, a bit higher than in a typical observed line, but the intensity of the synthetic line of $\mathrm{He}$ II $\lambda 4686$ roughly matches the observations. We have also computed for this model the IR He II $\lambda 10124$ line (4-5), which exhibits a symmetrical, strong emission. Vreux \& Andrillat (1979) estimated that the observed emission intensity ratio of $\lambda 10124$ to $\lambda 4686$ is around 1.3 for $\lambda$ Cep and $\zeta$ Pup. This ratio is also in agreement with our calculations.

The third model corresponds to a star with $T_{\text {eff }}=25,000$ $\mathrm{K}$ and $\log g=4.0$. This model produces a deep absorption profile in $\lambda 1640$ and emissions in $\lambda \lambda 4686$ and 3203 (Fig. $17 c$ ). This kind of profile roughly resembles the profile observed in some B[e] stars (Nota et al. 1996).

The fourth model is a CER one, with $T_{\text {eff }}=25,000 \mathrm{~K}$ and $\log g=3.5$. The profiles correspond to the line configuration observed in early B-type stars. The three lines are in absorption (Fig. 17d), in close accordance with the observed intensity ratios.

\section{CONCLUSIONS}

In high effective temperature models, the velocity regime dominates the profile features, while the strength of the line is strongly dependent on the surface gravity and the exophotospheric temperature distribution. The lower the gravity, the denser the wind and the stronger the emission rate. In Otype stars, the effect originated by the surface gravity is consistent with the stated luminosity criteria. This result indicates that He II $\lambda 4686$ can be used to derive the effective gravity.

From an observational point of view, line profiles in B stars present different characteristics from line profiles in $\mathrm{O}$ type stars. In general, our models reproduce these observations, but we also find some models for B-type stars with high-density winds that tend to produce strong emission 

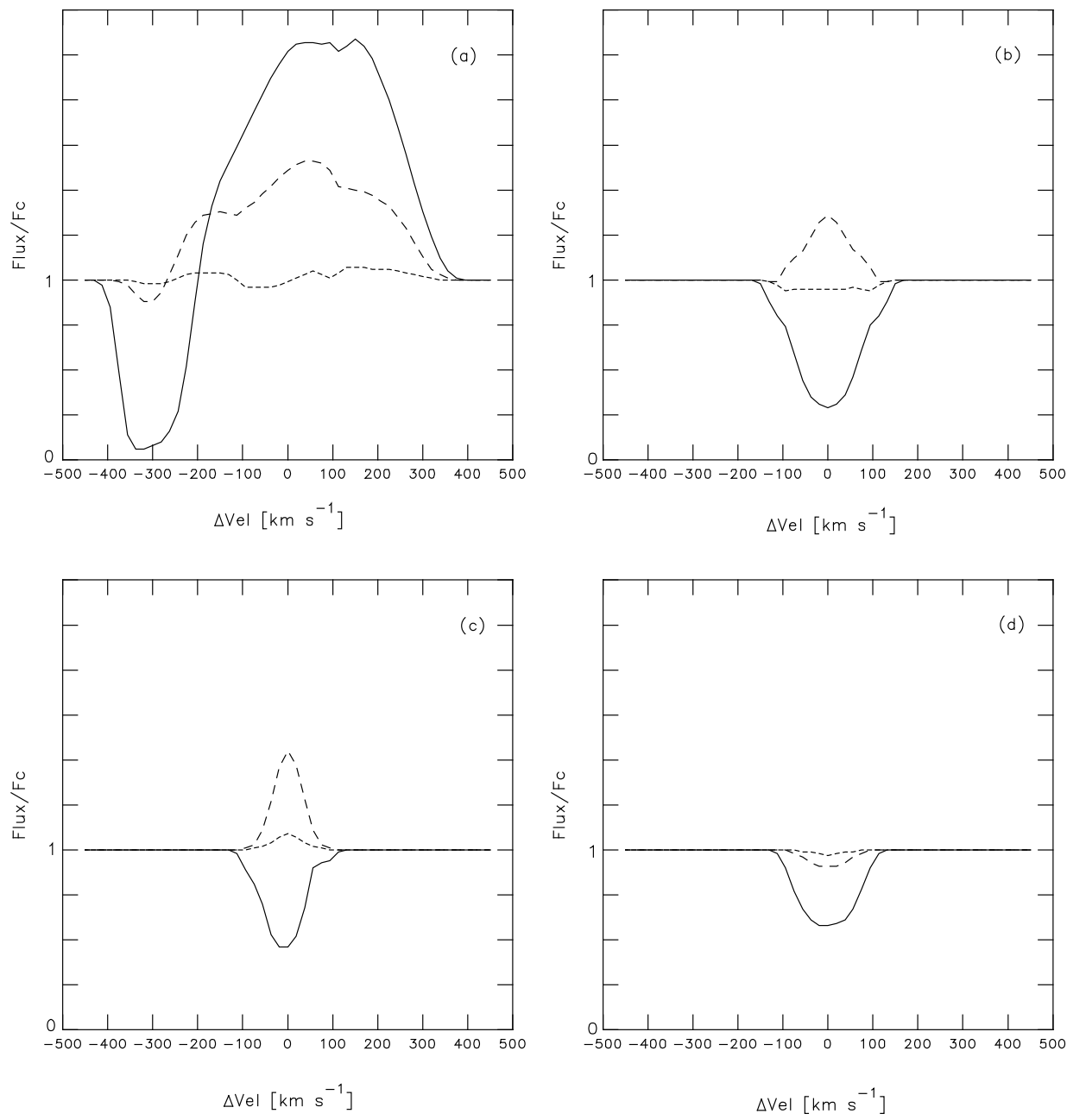

Fig. 17.-Line profiles of He II computed for models (a) HIR-19, with $T_{\text {eff }}=40,000 \mathrm{~K}, \log g=4.0, r_{f}=1.5 R_{\text {ph }} ;(b)$ HIR-22, with $T_{\text {eff }}=40,000 \mathrm{~K}$, $\log g=4.0, r_{f}=70 R_{\mathrm{ph}} ;(c)$ HER-3, with $T_{\text {eff }}=25,000 \mathrm{~K}, \log g=4.0, r_{f}=70 R_{\mathrm{ph}}$; and $(d)$ CER-2, with $T_{\mathrm{eff}}=25,000 \mathrm{~K}, \log g=3.5, r_{f}=1.5 R_{\mathrm{ph}}$. The line profiles are $\lambda 1640$ (solid line), $\lambda 4686$ (dashed line), and $\lambda 3203$ (dotted line). Other parameters of the models are given in Table 1.

and/or absorption line features that resemble, in some cases, the profiles of Of stars. This result would imply that He II reflects the physical state of an expanding hot wind (with $T>T_{\text {eff }}$ ) and therefore can mask the actual photospheric conditions, leading to an overestimate of the effective temperature.
We are grateful to N. Morrell, J. Sahade, and N. Walborn for their helpful comments and suggestions. We also wish to thank to our referee for critical comments that helped improve this work.
Andriesse, C. D. 1979, Ap\&SS, 61, 205

Auer, L., \& Mihalas, D. M. 1972, ApJS, 24, 193

Beals, C. S. 1951, Pub. Dom. Astrophys. Obs. Victoria, 9, 1

Cannon, C. J., \& Thomas, R. N. 1977, ApJ, 211, 910

Castor, J. I., Abbott, D. C., \& Klein, R. K. 1975, ApJ, 195, 157

Castor, J. I., \& Simon, T. 1983, ApJ, 265, 304

Catala, C., \& Kunasz, P. B. 1987, A\&A, 174, 158

Catala, C., Kunasz, P. B., \& Praderie, F. 1984, A\&A, 134, 402

Cidale, L. S., \& Ringuelet, A. E. 1993, ApJ, 411, 874

Cidale, L. S., Zorec, J., Maillard, J. P., \& Morrell, N. 2000, in IAU Colloq.

175, The Be Phenomenon in Early-Type Stars, ed. M. A. Smith, H. F.

Henrichs, \& J. Fabregat (ASP Conf. Ser. 214; San Francisco: ASP), 472

Conti, P. S., \& Leep, E. M. 1974, ApJ, 193, 113

Conti, P. S., \& Niemela, V. S. 1976, ApJ, 209, L37

Gabler, R., Gabler, A., Kudritzki, R.-P., Puls, J., \& Pauldrach, A. 1989, A\&A, 226, 162

Grady, C. A., Snow, T. P., Jr., \& Timothy, J. G. 1983, ApJ, 271, 691

Hamann, W.-R., \& Schmutz, W. 1987, A\&A, 174, 173

\section{REFERENCES}

Heap, S. R. 1971, A\&A, 15, 77

Herrero, A. 1987, A\&A, 186, 231

Herrero, A., Puls J., \& Villamariz, M. R. 2000, A\&A, 354, 193

Hillier, D. J. 1987, ApJS, 63, 947

Klein, R. I., \& Castor, J. I. 1978, ApJ, 220, 902

Kunasz, P. B. 1980, ApJ, 237, 819

Kurucz, R. L. 1979, ApJS, 40, 1

Mazzali, P. A. 1990, A\&A, 238, 191

Mihalas, D. M., \& Conti, P. S. 1980, ApJ, 235, 515

Mihalas, D. M., \& Kunasz, P. B. 1978, ApJ, 219, 635

Mihalas, D. M., \& Lockwood, G. W. 1972, ApJ, 175, 757

Morrison, N. D. 1975, ApJ, 202, 433

Nandy, K., Morgan, D. H., Willis, A. J., \& Gondhalekar, P. M. 1980, MNRAS, 193, 43P

Nota, A., Pasquali, A., Drissen, L., Leitherer, C., Robert, C., Moffat, A. F. J., \& Schmutz, W. 1996, ApJS, 102, 383

Pauldrach, A., Puls, J., \& Kudritzki, R.-P. 1986, A\&A, 164, 86

Peters, G. J. 1990, in Angular Momentum and Mass Loss for Hot Stars, ed. L. A. Willson \& R. Stalio (Dordrecht: Reidel), 219 
Petrenz, P., \& Puls, J. 2000, A\&A, 358, 956

Puls, J., et al. 1996, A\&A, 305, 171

Rauw, G., Morrison, N. D., Vreux, J.-M., Gosset, E., \& Mulliss, C. L. 2001, A\&A, 366, 585

Santolaya-Rey, A. E., Puls, J., \& Herrero, A. 1997, A\&A, 323, 488

Vázquez, A. C., Cidale, L. S., \& Ringuelet, A. E. 1993, ApJ, 419, 286
Venero, R. O. J., Cidale, L. S., \& Ringuelet, A. E. 1999, Rev. Mexicana Astron. Astrofis. Ser. Conf., 8, 127

Vreux, J.-M., \& Andrillat, Y. 1979, A\&A, 75, 93

Walborn, N. R. 1971, ApJS, 23, 257

Walborn, N. R., \& Nichols-Bohlin, J. 1987, PASP, 99, 40

Willis, A. J., \& Stickland, D. J. 1980, MNRAS, 190, 27P 